\title{
Multifunctional Role of Fas-Associated Death Domain Protein in Apoptosis
}

\author{
Key-Sun Kim* \\ Life Sciences Division, KIST, Cheongyang Box 131, Seoul 130-650, Korea
}

Received 12 November 2001

\section{Introduction}

The Fas-associated death domain protein (FADD) was discovered as a protein that interacts with the Fas receptor (Chinnaiyan et al., 1995; Zhang and Winoto, 1996). FADD consists of two distinct domains called the 'death effector domain' and the 'death domain' at its $\mathrm{N}$ - and C-terminus, respectively. The death domain of FADD (FADD-DD) interacts with the death domain of the Fas receptor, and the death effector domain of FADD (FADD-DED) interacts with the death effector domain of procaspase-8 (Boldin et al., 1996; Muzio et al., 1996). In Fas-mediated apoptosis, FADD is recruited to the Fas receptor, and recruits procaspase- 8 in turn. This results in the initiation of apoptotic processes (Medema et al., 1997; Muzio et al., 1998). However, FADD is also involved in T-cell proliferation (Zhang et al., 1998; Walsh et al., 1998 ) and necrosis (Kawahara et al., 1998; Matsumura et al., 2000), independent of known apoptosis mechanisms. In this article, the suggested roles of FADD in various pathways will be reviewed.

\section{FADD in apoptosis}

FADD is recruited by the Fas receptor upon the binding of the Fas ligand. It is known that the Fas ligand is a trimer, and induces the trimerization of receptors (Ashkenazi et al., 1998). The ligand-receptor complex structures of the tumor necrosis factor (TNF) receptor family show that two ligand monomers interact with a receptor, which indicates that the ligands play a critical role in the formation of the stable receptor trimer (Banner et al., 1993; Hymowitz et al., 1999; Cha et al., 2000). However, the association of receptors without ligand binding has been observed by the fluorescence energy transfer experiment. Furthermore, the apoptotic signal transduction is prevented if the pre-association domain is cleaved off the receptor, which indicates that pre-association is critical for the

*To whom correspondence should be addressed.

Tel: 82-2-958-5934; Fax: Tel: 82-2-958-5939

E-mail: keysun@kist.re.kr biological function. Chemical cross-linking of free receptors showed that the receptors exist as a trimer, even without the binding of the ligand. FADD was also observed in the chemically cross-linked receptor complex, but procaspase- 8 was not recruited nor activated (Siegel et al., 2000b). The chimeric receptor, which is generated by combining the extracellular domain of the platelet-derived growth factor (PDGF) and intracellular domain of TNF receptor I (TNFRI), was responsive to PDGF. The function that is elicited by PDGF by binding to the chimeric receptor was indistinguishable from that induced by the wild-type TNF receptor, even though PDGF induces the dimerization of receptors (Adam et al., 1995). This indicates that simple trimerization, or multimerization, may be insufficient to elicit a function, but a specific conformational change of the receptor is needed for the functional signal transduction. The assembly of Fas monomers into trimers without ligand is mediated by a pre-ligand assembly domain (PLAD) in the amino terminal region of Fas (Papoff et al., 1999; Siegel et al., 2000a). However, PLADs in the complex structure of TNFRI and TNF- $\beta$ have no interaction with one another (Banner et al., 1993). This indicates that either the ligand binding induces the conformational change of the receptor or pre-associated receptors interact with trimeric Fas ligand forming a supercluster. In any case, pre-association of the receptors is insufficient to induce an effective death-inducing signaling complex (DISC) until the trimeric ligand binds to the receptor. Interestingly, the extra-cellular domain of free TNFRI forms a dimer in the crystal structure (Naismith et al., 1996). The dimerization interface was far from the TNF binding region. This suggests the possible multimeric association by TNF ligation, but stoicheometry of chemical cross-linking of free receptors could not be explained.

Once FADD is recruited, procaspase- 8 or procaspase- 10 is recruited and activated by self-cleavage initiating apoptosis. The fact that free receptors already interact with FADD implies that a certain conformational change of FADD is necessary for efficient procasapse-8 recruitment. In the yeast two-hybrid system, FADD interacts with Fas-DD (Chinnaiyan et al., 1995) and procaspase-8 (Boldin et al., 1996; Muzio et 
al., 1996). If their respective binding affinities are the same, procaspase- 8 should be recruited to the free receptor, but procaspase- 8 is not recruited. This may indicate that Fas-DD and FADD are interacting in vivo, as observed in the yeast two-hybrid system. However, FADD and procaspae- 8 are not interacting strong enough unless the conformational change of FADD into a high affinity form is elicited, or an effective concentration of FADD is increased. Otherwise, the third molecule is interfering with FADD to interact with procaspase-8. An active caspase- 8 activates the executioner caspase such as caspase-3 that triggers various apoptotic processes (Ashkenazi et al., 1998), or activates BID to initiate a mitochondria-driven cell death (Li et al., 1998; Gross et al., 1999). The strong activation of caspase- 8 at DISC is observed in type I cells (BJAB). Only a small amount of DISC was formed in type II cells (Jurkat). Although two types of cell death are interconnected (Scaffidi et al., 1998; Scaffidi et al., 1999 b), caspase- 8 activated cell death is mainly observed in the type I cells, and mitochondria-driven cell death is predominantly observed in the type II cells.

In the TNF-induced apoptosis, FADD is recruited to TNFRI through the TNFRI-associated death domain protein (TRADD). TRADD recruits FADD, receptor-interacting protein (RIP), as well as the TNF receptor-associated factor 2 (TRAF2) to TNFRI, or to the death receptor 3 (DR3). The recruitment of FADD to TRADD induces caspase- 8 activation and apoptosis, while TRAF2 recruitment activates the NF- $\kappa B$ signal that leads to cell survival (Ashkenazi et al., 1998). The recruitment of RIP can activate both the apoptosis and NF- $\kappa \mathrm{B}$ signal. Once RIP is cleaved by caspase-8, the C-terminal domain of RIP enhances the caspase- 8 activation by enhancing the FADD recruitment to TRADD (Kim et al., 2000). FADD and RIP can also interact with each other through death domains (Grimm et al., 1996).

In the TNF-related apoptosis-inducing ligand (TRAIL)induced cell death, the death-associated protein 3 (DAP3) (Kissil et al., 1999 ) is reported to bridge the TRAIL receptor and FADD (Miyazaki et al., 2001). The TRAIL receptor binds to the N-terminus of DAP3, and FADD binds to the $\mathrm{C}$ terminus of DAP3. FADD-DED is shown to interact with the C-terminus of DAP3. DAP3 has a nucleotide-binding motif in the N-terminal end. The binding of GTP to the nucleotidebinding motif enhances the recruitment of FADD, and the subsequent caspase- 8 activation. Interestingly, DAP3 was also observed in DISC that was induced by the Fas ligand. The Fas receptor and DAP3 do not interact directly, but FADD interacts with DAP3. This indicates that DAP3 is recruited to the Fas receptor via FADD. However, there is a conflicting report showing that DAP3 is not a member of DISC (Kissil et al., 1999). FADD-DED binds to both DAP3 and procasapse8 , but it is unknown whether or not DAP3 enhances the association of procasaspe- 8 with FADD. The death effector domain of FADD or procaspase- 8 also interacts with the FADD-like IL-1 $\beta$-converting enzyme (FLICE)-inhibitory proteins (FLIPs). The FLIP is known to regulate the apoptosis by interfering with the activation of caspase-8 (Irmler et al., 1997; Scaffidi et al., 1999a).

\section{FADD in necrosis}

Fas-mediated apoptosis is initiated by caspase- 8 activation, so casapse- 8 is indispensable for the Fas-mediated apoptosis. However, oligomerization of FADD could kill Jurkat cells that are deficient in caspase-8, or the wild-type cells that were treated with the broad-spectrum caspase inhibitor. The dying Jurkat cells by this pathway showed a necrotic morphological change (Kawahara et al., 1998; Matsumura et al., 2000). It is also reported that FADD-DED kills $E$. coli by inducing reactive oxygen species (Lee et al., 2000). These results imply that the death effector domain is related to necrotic cell death. Artificial oligomerization, using the FK506 binding protein fusion (Nagata group), showed that FADD-DED is responsible for the FADD-mediated necrotic cell death. The oligomerization of the death-effector domain, but not the death domain of FADD, was able to induce necrosis in caspase- 8 deficient cells. This process was accompanied by a loss of mitochondrial membrane potential, but not by the release of cytochrome $\mathrm{c}$ from mitochondria. The reduction of membrane potential can be inhibited by pyrolidine dithiocarbamate, a metal chelator and antioxidant (Matsumura et al., 2000). The killing of E. coli by FADD-DED was also alleviated by adding antioxidants, which indicates that a similar pathway exists in E. coli (Lee et al., 2000). The broadspectrum caspase inhibitor, zVAD-fmk, only weakly blocked cell death in the resting $\mathrm{T}$ cells, and had no effect on activated $\mathrm{T}$ cells in the FasL-induced cell death. This suggests that the FasL-induced cell death of the activated $\mathrm{T}$ cell is caspaseindependent (Holler et al., 2000). The caspase-independent cell death showed the necrotic morphological change, and is completely resistant in FADD-deficient cells. The necrotic cell death is also completely resistant in RIP-deficient, or in the cells that have defective RIP activity. This indicates that the RIP kinase activity is essential for the necrotic cell death. In FasL- and TRAIL-induced necrotic cell death, FADD-DED is required, but FADD is not required in the TNF-induced necrotic cell death. It is interesting that RIP was directly recruited to Fas in the FADD-deficient cell, although the necrotic cell death is not observed. The authors, therefore, suggest that an unidentified protein is recruited by FADD and phosphorylated by the receptor-bound kinase RIP in order to initiate necrotic cell death. However, further work is necessary to explain why FADD is not required in the TNF-induced necrotic cell death if indeed FADD plays such a critical role in recruiting another factor by the death effector domain. In the TNF-induced apoptosis, no protein other than FADD is known to have the death effector domain.

\section{FADD in cell proliferation}

FADD-deficient mice are embryonic lethal with heart 
developmental problems, and die around 10 days after gestation (Zhang et al., 1998). The inactivation of FADD by the expression of a FADD dominant negative molecule, or by gene targeting, leads to the impairment of activation-induced T-cell proliferation. This suggests a role for FADD in T-cell development and activation, as well as in embryonic development (Newton et al., 1998; Walsh et al., 1998). In pursuing the role of FADD in T-cell development, two forms of FADD were identified. It was discovered that one of them was phosphorylated at serine 194 , but it was unrelated to the Fas-mediated apoptosis. The phosphorylation of FADD that correlated with the cell cycle, and a $70-\mathrm{kDa}$ cell-cycleregulated kinase that specifically binds to the $\mathrm{C}$-terminal half of FADD, was identified. Because Fas-mediated apoptosis is independent of the cell cycle, it is possible that FADD regulates proliferation through its interaction with the cell cycle-regulated kinase (Scaffidi et al., 1998). In the absence of FADD, cell cycle abnormality was reported (Zhang et al., 2001).

FADD is also involved in acid sphingomyelinase activation (Adam-Klages et al., 1998; Schwandner et al., 1998). Activation of acid sphingomyelinase releases ceramide, a metabolite that triggers cell death when administered exogenously. Stimulation of Fas blocks T lymphocyte calcium channels through sphingomyelinase and sphingolipids (Lepple-Wienhues et al., 1999; Hueber et al., 2000). FADD is required for the activation of sphingomyelinase, but the overexpression of caspase- 8 does not lead to enhanced acid sphingomyelinase activation. This implies that the activation of sphingomyelinase is different from the apoptotic pathways.
However, broad-spectrum caspase inhibitors reduce the sphingomyelinase activity (Schwandner et al., 1998). An influx of calcium ions into the $\mathrm{T}$ cell is needed to activate the $\mathrm{T}$ cell, and allow it to proliferate in response to the antigen. However, a dominant negative mutant of FADD (FADD-DN) inhibits proliferation and leads to impaired calcium mobilization in both T-cells and fibroblasts, which indicates that the effect of FADD-DN on proliferation and calcium mobilization is not T-cell specific (Hueber et al., 2000). Blocking the FADD function by FADD-DN or FADDdeficient cells affected the cell cycle, and prevented its entry into the $\mathrm{S}$ phase of $\mathrm{T}$ cells. However, the regulation of cell cycle proteins appears to be different in the FADD-deficient cell (Zhang et al., 2001), and in the FADD-DN transgenic T cell (Hueber et al., 2000). Also, blocking the FADD function by FADD-DN could cause lymphoid malignancy, indicating that FADD represents a tumor suppressor (Newton et al., 2000).

\section{Structural basis of multiple functions of FADD}

FADD is a 208 amino acid long protein that consists of two domains-the death effector domain at its $\mathrm{N}$-terminus and the death domain at its $\mathrm{C}$-terminus. In addition to these two domains, the peptide of about 20 amino acids is in the Cterminal end, which is the first degraded in the protease digestion, indicating that the C-terminal peptide is not structured (Lee et al., 2000). Ser ${ }^{194}$ in the C-terminal peptide is phosphorylated by the cell-cycle-regulated kinase (Fig. 1). Although the phosphorylation of FADD correlates with the

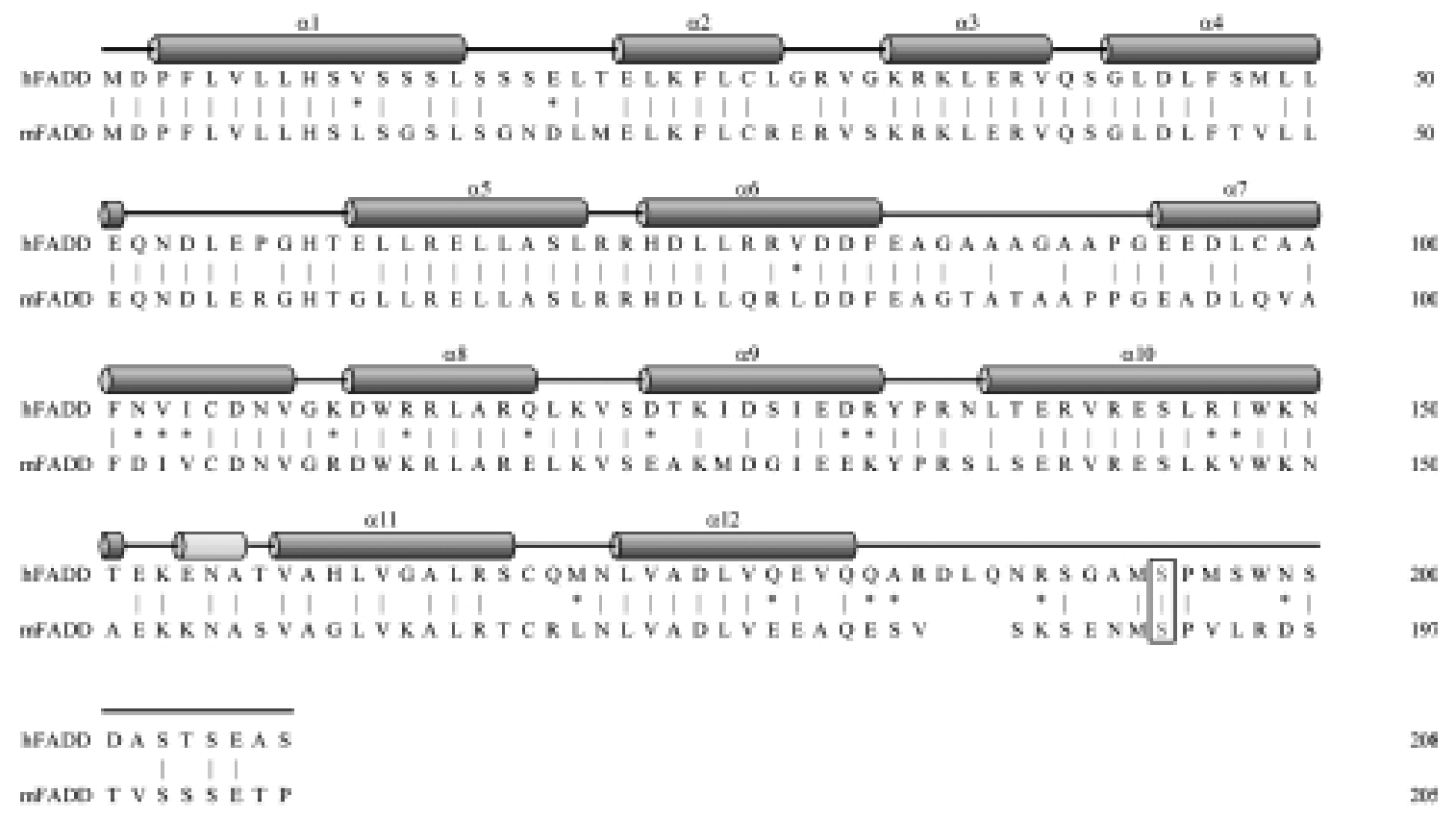

Fig. 1. Sequences of human and mouse FADD. Secondary structures of FADD are from the structure of human FADD-DED (M $\left.{ }^{1}-E^{83}\right)$ and from the structure of mouse FADD-DD $\left(\mathrm{A}^{89}-\mathrm{S}^{183}\right)$. The helix number is given, assuming the full length FADD. The structure of each domain has 6 helices in a determined structure. The phosphorylation site ( $\mathrm{S}^{194}$ of human FADD) of FADD is shown in red. A $3^{10}$ helix is located between $\alpha 10$ and $\alpha 11$ helix. 

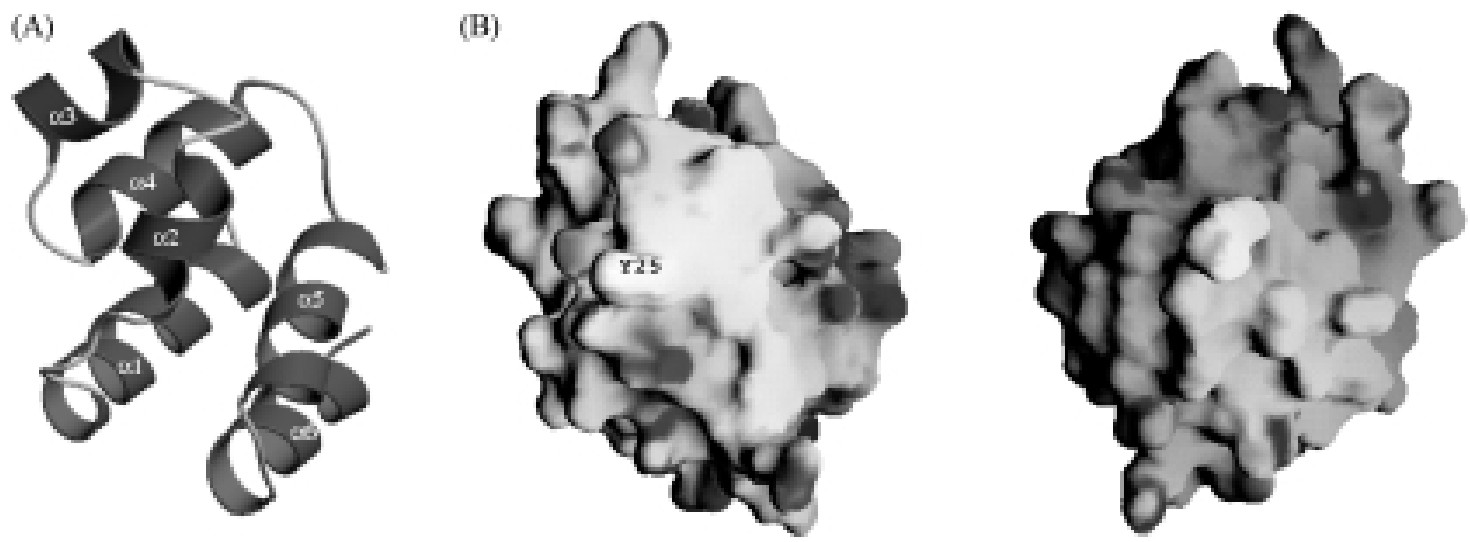

Fig. 2. (A) Ribbon drawing of the human FADD-DED F25Y. (B) The electrostatic potential surface of FADD-DED is shown in the same view as in A, and the functionally critical residue Tyr25 is labeled (left). Residue 25 is Phe in the wild-type protein. The opposite side of the hydrophobic surface is shown at the right. The negatively-charged surface is in red, and the positively-charged surface is in blue. The hydrophobic surface is shown in white (Eberstadt et al., 1998).
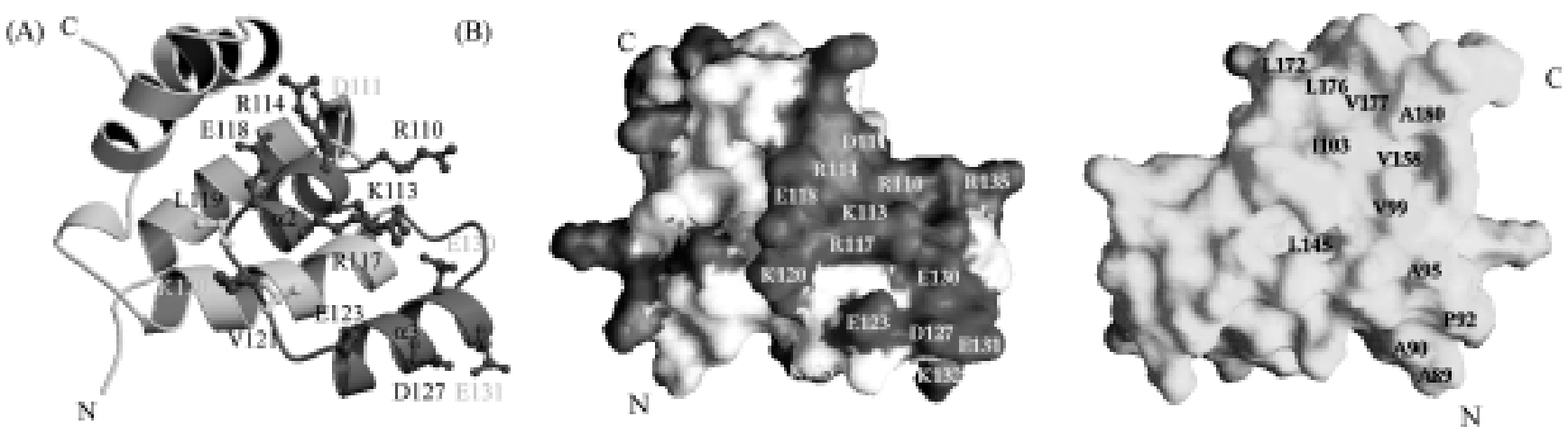

Fig. 3. (A) A ribbon drawing of the mouse FADD-DD with side chains in the suggested interaction site. (B) The electrostatic potential surface of FADD-DD is shown in the same view as in A (left). The colors of the potential surface are the same as in Figure 2. The hydrophobic residues on the opposite side are shown in sky blue (right) (adapted from Jeong et al., 1999)

cell cycle, an additional function of the phosphorylated FADD has not yet been identified (Scaffidi et al., 2000). The phosphorylation of FADD may affect the binding of FADD to DISC, or recruit another factor that is related to other functions of FADD such as cell proliferation.

The structure of FADD-DED consists of $6 \alpha$-helices that are arranged in an anti-parallel fashion, and it has two hydrophobic patches on the surface (Eberstadt et al., 1998). The hydrophobic residues from one of these sites are highly conserved among the death-effector domains of procasaspe- 8 and FLIPs. Phe 25 in the $\alpha 2$ helix, which is located in the middle of this hydrophobic site, is critical for the apoptotic activity and binding to procaspase- 8 . The substitution of Phe25 by glycine inactivated FADD in apoptosis, but the substitution by tyrosine or tryptophan had little effect on apoptosis and binding to procasapse-8. This indicates that the hydrophobic interaction plays a critical role in the function. However, the other hydrophobic patch is not well characterized, although it may not be critical for apoptotic activity. The interaction site with procasapse- 8 appears to be a hydrophobic surface that contains Phe25, and the same site seems to be used in an interaction with FLIP (Fig. 2). However, the interaction site of FADD-DED with DAP3 would be different from the binding site of procaspase- 8 since DAP3 and procasapse- 8 should bind simultaneously to FADD-DED in the TRAIL-induced apoptosis (Miyazaki et al., 2001). In addition, the unaccounted interaction of FADDDED in necrotic apoptosis could be different from the binding site of procaspase- 8 .

The structure FADD-DD also consists of $6 \alpha$-helices that are arranged in an anti-parallel fashion that is similar to the death-effector domain (Jeong et al., 1999). The surface of the death domain consists of the hydrophobic and hydrophilic surface. It is known that the hydrophilic surface plays a critical role in protein interaction, and the hydrophobic surface may be involved in domain interaction, which could play a role in the conformational change in signal transduction (Fig. 3). The helices $\alpha 2$ and $\alpha 3$ are involved in the interaction with Fas-DD, but the exact interaction mode is controversial (Jeong et al., 1999; Weber et al., 2001a,b). The complex 
structure of the death domains of Pelle/Tube (Xiao et al., 1999), and the caspase recruitment domain of Apaf-1/ procaspase-9 (Qin et al., 1999), were determined. However, the binding sites that are observed in the complex structure were different in each complex. The interaction site of Apaf-1 is similar to the suggested binding site of Fas-DD and FADD$\mathrm{DD}$, but those of other domains in the complex are different from the suggested binding sites of Fas-DD and FADD-DD. From the observed binding sites in the domain, a heterohexameric complex of Fas-DD and FADD-DD was proposed (Weber et al., 2001a,b). In the proposed complex, either a Fas trimer or a FADD trimer is located at the center, which is surrounded by three FADD or Fas molecules in analogy to the TNF- $\beta$ /TNFRI complex structure (Banner et al., 1993). The Fas ligand and Fas receptor would form at least a hexamer, but they may form a super-cluster. Considering that the dimerization of TNFRI also effectively transduced the signals (Adam et al., 1995), it appears that the conformational change of Fas is required for the effective recruitment of FADD and activation of caspase-8. On the other hand, various binding patterns of the domain may be crucial for the multiple functions that are implicated for FADD. FADD may be involved in many functions without participating in DISC, but directly binding to other molecules. FADD mediates Fas-, TNF- and TRAIL-induced apoptosis. In TRAIL-induced apoptosis, it is unclear yet whether or not FADD-DD plays a role in the signal transduction. However, the binding surface of the FADD death domain plays a critical role in both the Fas- and TNF-mediated apoptosis, which indicates that the Fas- and TNF-induced apoptosis use the same binding surface of FADD to recruit procasapse- 8 (Bang et al., 2000). This indicates that different signaling molecules use a similar structural interaction to trigger the same cellular event in the convergent signal transduction.

\section{Concluding Remarks}

FADD is involved in many cellular processes. FADD could get involved in various cellular processes either by participating in the DISC formation, or by directly interacting with other molecules. Some functions may operate as a main process in the cell, but others may be operational only under special circumstances. Some functions require the conformational change of FADD, but others may need the FADD molecule itself to elicit functions. Sorting out all of the functions will be pivotal in understanding the complexity of the cellular signal transduction that is related to FADD.

\section{References}

Adam, D., Kessler, U. and Kronke, M. (1995) Cross-linking of the p55 tumor necrosis factor receptor cytoplasmic domain by a dimeric ligand induces nuclear factor-kappa B and mediates cell death. J. Biol. Chem. 270, 17482-17487.

Adam-Klages, S., Schwandner, R., Adam, D., Kreder, D.,
Bernardo, K. and Kronke, M. (1998) Distinct adapter proteins mediate acid versus neutral sphingomyelinase activation through the p55 receptor for tumor necrosis factor. J. Leukoc. Biol. 63, 678-682.

Ashkenazi, A. and Dixit, V. M. (1998) Death receptors: signaling and modulation. Science 281, 1305-1308.

Bang, S., Jeong, E. -J., Kim, I. -K., Jung, Y. -K. and Kim, K.-S. (2000) Fas- and tumor necrosis factor-mediated apoptosis uses the same binding surface of FADD to trigger signal transduction. A typical model for convergent signal transduction. J. Biol. Chem. 275, 36217-36222.

Banner, D. W., D’Arcy, A., Janes, W., Gentz, R., Schoenfeld, H. J., Broger, C., Loetscher, H. and Lesslauer, W. (1993) Crystal structure of the soluble human $55 \mathrm{kd}$ TNF receptor-human TNF beta complex: implications for TNF receptor activation. Cell 73, 431-445.

Boldin, M. P., Goncharov, T. M., Goltsev, Y. V. and Wallach, D. (1996) Involvement of MACH, a novel MORT1/FADDinteracting protease, in Fas/APO-1- and TNF receptor-induced cell death. Cell 85, 803-815.

Cha, S. S., Sung, B. J., Kim, Y. A., Song, Y. L., Kim, H. J., Kim, S., Lee, M. S. and Oh, B. H. (2000) Crystal structure of TRAIL-DR5 complex identifies a critical role of the unique frame insertion in conferring recognition specificity. J. Biol. Chem. 275, 31171-31177.

Chinnaiyan, A. M., O'Rourke, K., Tewari, M. and Dixit, V. M. (1995) FADD, a novel death domain-containing protein, interacts with the death domain of Fas and initiates apoptosis. Cell 81, 505-512.

Eberstadt, M., Huang, B., Chen, Z., Meadows, R. P., Ng, S. C., Zheng, L., Lenardo, M. J. and Fesik, S. W. (1998) NMR structure and mutagenesis of the FADD (Mort1) death-effector domain. Nature 392, 941-945.

Grimm, S., Stanger, B. Z. and Leder, P. (1996) RIP and FADD: two "death domain"-containing proteins can induce apoptosis by convergent, but dissociable, pathways. Proc. Natl. Acad. Sci. USA 93, 10923-10927.

Gross, A., Yin, X. M., Wang, K., Wei, M. C., Jockel, J., Milliman, C., Erdjument-Bromage, H., Tempst, P. and Korsmeyer, S. J. (1999) Caspase cleaved BID targets mitochondria and is required for cytochrome $\mathrm{c}$ release, while BCL-XL prevents this release but not tumor necrosis factor-R1/Fas death. J. Biol. Chem. 274, 1156-1163.

Holler, N., Zaru, R., Micheau, O., Thome, M., Attinger, A., Valitutti, S., Bodmer, J. L., Schneider, P., Seed, B. and Tschopp, J. (2000) Fas triggers an alternative, caspase-8independent cell death pathway using the kinase RIP as effector molecule. Nat. Immunol. 1, 489-495.

Hueber, A. O., Zornig, M., Bernard, A. M., Chautan, M. and Evan, G. (2000) A dominant negative Fas-associated death domain protein mutant inhibits proliferation and leads to impaired calcium mobilization in both T- cells and fibroblasts. J. Biol. Chem. 275, 10453-10462.

Hymowitz, S. G., Christinger, H. W., Fuh, G., Ultsch, M., O'Connell, M., Kelley, R. F., Ashkenazi, A. and de Vos, A. M. (1999) Triggering cell death: the crystal structure of Apo2L/ TRAIL in a complex with death receptor 5. Mol. Cell. 4, 563571.

Irmler, M., Thome, M., Hahne, M., Schneider, P., Hofmann, K., Steiner, V., Bodmer, J. L., Schroter, M., Burns, K., Mattmann, 
C., Rimoldi, D., French, L. E. and Tschopp, J. (1997) Inhibition of death receptor signals by cellular FLIP. Nature 388, 190-195.

Jeong, E. -J., Bang, S., Lee, T. H., Park, Y. I., Sim, W. -S. and Kim, K.-S. (1999) The solution structure of FADD death domain. Structural basis of death domain interactions of Fas and FADD. J. Biol. Chem. 274, 16337-16342.

Kawahara, A., Ohsawa, Y., Matsumura, H., Uchiyama, Y. and Nagata, S. (1998) Caspase-independent cell killing by Fasassociated protein with death domain. J. Cell. Biol. 143, 13531360.

Kim, J. W., Choi, E. J. and Joe, C. O. (2000) Activation of deathinducing signaling complex (DISC) by pro-apoptotic C-terminal fragment of RIP. Oncogene 19, 4491-4499.

Kissil, J. L., Cohen, O., Raveh, T. and Kimchi, A. (1999) Structure-function analysis of an evolutionary conserved protein, DAP3, which mediates TNF-alpha- and Fas-induced cell death. EMBO J. 18, 353-362.

Lee, S. W., Ko, Y. G., Bang, S., Kim, K.-S. amd Kim, S. (2000) Death effector domain of a mammalian apoptosis mediator, FADD, induces bacterial cell death. Mol. Microbiol. 35, 15401549.

Lepple-Wienhues, A., Belka, C., Laun, T., Jekle, A., Walter, B., Wieland, U., Welz, M., Heil, L., Kun, J., Busch, G., Weller, M., Bamberg, M., Gulbins, E. and Lang, F. (1999) Stimulation of CD95 (Fas) blocks T lymphocyte calcium channels through sphingomyelinase and sphingolipids. Proc. Natl. Acad. Sci. USA 96, 13795-13800.

Li, H., Zhu, H., Xu, C. J. and Yuan, J. (1998) Cleavage of BID by caspase 8 mediates the mitochondrial damage in the Fas pathway of apoptosis. Cell 94, 491-501.

Matsumura, H., Shimizu, Y., Ohsawa, Y., Kawahara, A., Uchiyama, Y. and Nagata, S. (2000) Necrotic death pathway in Fas receptor signaling. J. Cell Biol. 151, 1247-1256.

Medema, J. P., Scaffidi, C., Kischkel, F. C., Shevchenko, A., Mann, M., Krammer, P. H. and Peter, M. E. (1997) FLICE is activated by association with the CD95 death-inducing signaling complex (DISC). EMBO J. 16, 2794-2804.

Miyazaki, T. and Reed, J. C. (2001) A GTP-binding adapter protein couples TRAIL receptors to apoptosis- inducing proteins. Nat. Immunol. 2, 493-500.

Muzio, M., Chinnaiyan, A. M., Kischkel, F. C., O'Rourke, K., Shevchenko, A., Ni, J., Scaffidi, C., Bretz, J. D., Zhang, M., Gentz, R., Mann, M., Krammer, P. H., Peter, M. E. and Dixit, V. M. (1996) FLICE, a novel FADD-homologous ICE/CED-3like protease, is recruited to the CD95 (Fas/APO-1) death-inducing signaling complex. Cell 85, 817-827.

Muzio, M., Stockwell, B. R., Stennicke, H. R., Salvesen, G. S. and Dixit, V. M. (1998) An induced proximity model for caspase-8 activation. J. Biol. Chem. 273, 2926-2930.

Naismith, J. H., Devine, T. Q., Kohno, T. and Sprang, S. R. (1996) Structures of the extracellular domain of the type I tumor necrosis factor receptor. Structure 4, 1251-1262.

Newton, K., Harris, A. W., Bath, M. L., Smith, K. G. and Strasser, A. (1998) A dominant interfering mutant of FADD/ MORT1 enhances deletion of autoreactive thymocytes and inhibits proliferation of mature T lymphocytes. EMBO J. 17, 706-718.

Newton, K., Harris, A. W. and Strasser, A. (2000) FADD/MORT1 regulates the pre-TCR checkpoint and can function as a tumour suppressor. EMBO J. 19, 931-941.

Papoff, G., Hausler, P., Eramo, A., Pagano, M. G., Di Leve, G., Signore, A. and Ruberti, G. (1999) Identification and characterization of a ligand-independent oligomerization domain in the extracellular region of the CD95 death receptor. J. Biol. Chem. 274, 38241-38250.

Qin, H., Srinivasula, S. M., Wu, G., Fernandes-Alnemri, T., Alnemri, E. S. and Shi, Y. (1999) Structural basis of procaspase- 9 recruitment by the apoptotic protease- activating factor 1. Nature 399, 549-557.

Scaffidi, C., Fulda, S., Srinivasan, A., Friesen, C., Li, F., Tomaselli, K. J., Debatin, K. M., Krammer, P. H. and Peter, M. E. (1998) Two CD95 (APO-1/Fas) signaling pathways. EMBO J. 17, 1675-1687.

Scaffidi, C., Schmitz, I., Krammer, P. H. and Peter, M. E. (1999a) The role of c-FLIP in modulation of CD95-induced apoptosis. J. Biol. Chem. 274, 1541-1548.

Scaffidi, C., Schmitz, I., Zha, J., Korsmeyer, S. J., Krammer, P. H. and Peter, M. E. (1999b) Differential modulation of apoptosis sensitivity in CD95 type I and type II cells. J. Biol. Chem. 274, 22532-22538.

Scaffidi, C., Volkland, J., Blomberg, I., Hoffmann, I., Krammer, P. H. and Peter, M. E. (2000) Phosphorylation of FADD/ MORT1 at serine 194 and association with a 70- $\mathrm{kDa}$ cell cycleregulated protein kinase. J. Immunol. 164, 1236-1242.

Schwandner, R., Wiegmann, K., Bernardo, K., Kreder, D. and Kronke, M. (1998) TNF receptor death domain-associated proteins TRADD and FADD signal activation of acid sphingomyelinase. J. Biol. Chem. 273, 5916-5922.

Siegel, R. M., Chan, F. K., Chun, H. J. and Lenardo, M. J. (2000a) The multifaceted role of Fas signaling in immune cell homeostasis and autoimmunity. Nat. Immunol. 1, 469-474.

Siegel, R. M., Frederiksen, J. K., Zacharias, D. A., Chan, F. K., Johnson, M., Lynch, D., Tsien, R. Y. and Lenardo, M. J. (2000b) Fas preassociation required for apoptosis signaling and dominant inhibition by pathogenic mutations. Science $\mathbf{2 8 8}$, 2354-2357.

Walsh, C. M., Wen, B. G., Chinnaiyan, A. M., O'Rourke, K., Dixit, V. M. and Hedrick, S. M. (1998) A role for FADD in T cell activation and development. Immunity 8, 439-449.

Weber, C. H. and Vincenz, C. (2001a) The death domain superfamily: a tale of two interfaces? Trends Biochem. Sci. 26, 475-481.

Weber, C. H. and Vincenz, C. (2001b) A docking model of key components of the DISC complex: death domain superfamily interactions redefined. FEBS Lett 492, 171-176.

Xiao, T., Towb, P., Wasserman, S. A. and Sprang, S. R. (1999) Three-dimensional structure of a complex between the death domains of Pelle and Tube. Cell 99, 545-555.

Zhang, J., Cado, D., Chen, A., Kabra, N. H. and Winoto, A. (1998) Fas-mediated apoptosis and activation-induced T-cell proliferation are defective in mice lacking FADD/Mort1. Nature 392, 296-300.

Zhang, J., Kabra, N. H., Cado, D., Kang, C. and Winoto, A. (2001) FADD-deficient $\mathrm{T}$ cells exhibit a disaccord in regulation of the cell cycle machinery. J. Biol. Chem. 276, 29815-29818.

Zhang, J. and Winoto, A. (1996) A mouse Fas-associated protein with homology to the human Mort1/FADD protein is essential for Fas-induced apoptosis. Mol. Cell. Biol. 16, 2756-2763. 